PROCEEDINGS OF THE

AMERICAN MATHEMATICAL SOCIETY

Volume 131, Number 10, Pages 3059-3062

S 0002-9939(03)07005-9

Article electronically published on March 17, 2003

\title{
ALMOST PERIODIC SETS AND SUBACTIONS IN TOPOLOGICAL DYNAMICS
}

\author{
ETHAN AKIN AND JOSEPH AUSLANDER
}

(Communicated by Michael Handel)

\begin{abstract}
Let $\Gamma$ be a group with subgroup $\Lambda$. We show, under certain conditions, that an almost periodic point for the action of $\Lambda$ is also almost periodic for $\Gamma$. This is applied to obtain a theorem of Glasner.
\end{abstract}

We will be considering flows $(X, S)$ where $X$ is a compact Hausdorff space and $S$ is a semigroup. In many cases of interest to us, the elements of $S$ define homeomorphisms of $X$.

The point $x \in X$ is said to be an almost periodic point of $(X, S)$ if $\overline{x S}$ is a minimal set and $x \in \overline{x S}$.

The following elementary lemma will be used frequently in the sequel.

Lemma 1. Let $(X, S)$ and $(Y, S)$ be flows, let $x \in X$ be almost periodic, and let $y \in Y$. Then there is an almost periodic point of the form $\left(x, y^{\prime}\right) \in \overline{(x, y) S}$.

Proof. Let $\left(x_{1}, y_{1}\right) \in \overline{(x, y) S}$ be almost periodic. Let $\left\{s_{i}\right\}$ be a net in $S$ such that $x_{1} s_{i} \rightarrow x$. Then (a subnet of) $\left(x_{1}, y_{1}\right) s_{i} \rightarrow\left(x, y^{\prime}\right)$ for some $y^{\prime}$. Since $\left(x, y^{\prime}\right)$ is in the orbit closure of an almost periodic point, it is almost periodic.

An almost periodic set for $(X, S)$ is a subset $A$ of $X$ such that if $z \in X^{|A|}$ with range $(z)=A$, then $z$ is an almost periodic point of the flow $\left(X^{|A|}, S\right)$. $(|A|$ denotes the cardinality of $A$.) If $x \in X$ is an almost periodic point, then it is easy to see that there is a maximal almost periodic set which contains $x$.

The following lemma lists elementary properties of almost periodic sets.

Lemma 2. (i) Any non-empty subset of an almost periodic set is an almost periodic set. (In particular, every point of an almost periodic set is almost periodic.)

(ii) Let $A$ be a maximal almost periodic set for $(X, S)$, and let $x \in X$. Then there is an $x^{\prime} \in A \cap \overline{x S}$ such that $x$ and $x^{\prime}$ are proximal. (That is, if $W$ is any neighborhood of the diagonal $\Delta$, then there is an $s \in S$ such that $\left(x s, x^{\prime} s\right) \in W$.)

(iii) Let $\pi:(X, S) \rightarrow(Y, S)$ be an onto flow homomorphism, and let $B$ be an almost periodic set in $Y$. Then there is an almost periodic set $A$ in $X$ such that $\pi(A)=B$.

(iv) If $A$ is a maximal almost periodic set in $X$, then $\pi(A)$ is a maximal almost periodic set in $Y$.

$(v)$ Suppose range $(z)$ is a maximal almost periodic set. Let $z^{\prime} \in \overline{z S}$. Then range $\left(z^{\prime}\right)$ is a maximal almost periodic set.

Received by the editors April 12, 2002.

2000 Mathematics Subject Classification. Primary 37B05. 
Proof. (i) follows from the well known (and easily proved) fact that a homomorphic image of an almost periodic point is almost periodic.

(ii) Let $z \in X^{|A|}$ such that range $(z)=A$, and let $\left(z, x^{\prime}\right) \in \overline{(z, x) S}$ be an almost periodic point. Let $\left\{s_{i}\right\}$ be a net in $S$ with $(z, x) s_{i} \rightarrow\left(z, x^{\prime}\right)$. Since $A=\operatorname{range}(z)$ is maximal, $x^{\prime} \in A$, so we have $\left(x^{\prime}, x\right) s_{i} \rightarrow\left(x^{\prime}, x^{\prime}\right)$.

(iii) Let $w \in Y^{|B|}$ with range $(w)=B$. Then $w$ is an almost periodic point in $\left(Y^{|B|}, S\right)$. Let $z$ be an almost periodic point in $X^{|B|}$ with $\pi(z)=w$, and let $A=\operatorname{range}(z)$. Then $A$ is an almost periodic set and $\pi(A)=B$.

(iv) follows immediately from (iii).

$(v)$ Clearly range $\left(z^{\prime}\right)$ is an almost periodic set. Suppose $\left(z^{\prime}, x^{\prime}\right)$ is an almost periodic point. Let $\left\{s_{i}\right\}$ be a net such that $\left(z^{\prime}, x^{\prime}\right) s_{i} \rightarrow(z, x)$. Since $(z, x)$ is almost periodic, and range $(z)$ is maximal, $x \in \operatorname{range}(z)$. It follows that $x^{\prime} \in \operatorname{range}\left(z^{\prime}\right)$. (If not, there would be a $y^{\prime} \in \operatorname{range}\left(z^{\prime}\right)$ with $x^{\prime}$ and $y^{\prime}$ proximal and $x^{\prime} \neq y^{\prime}$ which would contradict $\left(x^{\prime}, y^{\prime}\right)$ almost periodic.)

Let $(X, S)$ be a flow. Then $x \in X$ is said to be a distal point if for every $y \in \overline{x S}$ with $y \neq x, x$ and $y$ are distal (not proximal). A minimal flow is called point distal if it contains a distal point.

Corollary 1. Let $x$ be a distal point for a flow $(X, S)$. Then $x$ is an almost periodic point.

Proof. This follows from (ii) of Lemma 2.

Let $\Gamma$ be a group which acts minimally on a space $X$, and let $S$ be a subsemigroup of $\Gamma$ which generates $\Gamma$. It is not in general the case that $(X, S)$ is minimal. (An example is given at the end of the paper.) Two cases where $(X, S)$ is in fact minimal follow.

Lemma 3. Let $(X, \Gamma)$ be a minimal flow, with $\Gamma$ an abelian group. Let $S$ be a subsemigroup of $\Gamma$ which generates $\Gamma$ (equivalently $\left.\Gamma=S S^{-1}\right)$. Then $(X, S)$ is minimal.

Proof. Let $X_{0}$ be a minimal set for $S$. Let $x \in X_{0}$ and let $s \in S$. Then $x s^{-1}$ is an almost periodic point for $S$, so $x s^{-1}$ is in $X_{1}$, an $S$ minimal set. Then $x=x s^{-1} s \in X_{1}$ so $X_{1}=X_{0}$. Therefore $x S^{-1} \subset X_{0}$, and it follows that $x \Gamma \subset X_{0}$, so $X_{0}=X$.

Theorem 1. Let $\Gamma$ be a group and let $S$ be a semigroup which generates $\Gamma$. Let $(X, \Gamma)$ be a point distal minimal flow. Then $(X, S)$ is minimal.

Proof. Let $x$ be a distal point for $(X, \Gamma)$, and let $X_{0}=\overline{x S}$. It follows from Corollary 1 that $X_{0}$ is an $S$ minimal set. It is sufficient to show that $x \Gamma \subset X_{0}$. For this, in turn, it is sufficient to show that $x s \sigma^{-1} \in X_{0}$, for $\sigma, s \in S$. (Note that $x s \sigma^{-1}$ is also a distal point, and each $t \in \Gamma$ is a finite product of elements of $S$ and $S^{-1}$.) Clearly $x \sigma \in X_{0}$. Let $\Gamma_{1}$ be the cyclic subgroup generated by $s$, and let $S_{1}$ be the semigroup generated by $s$. Now $x \sigma$ is a distal point, hence an almost periodic point for $\left(X, \Gamma_{1}\right)$. By Lemma 3 we have $x \sigma s^{-1} \in \overline{x \sigma \Gamma_{1}}=\overline{x \sigma S_{1}} \subset \overline{x S}=X_{0}$.

Lemma 4. Let $\Gamma$ be a group, and let $S$ be a subsemigroup of $\Gamma$ which generates $\Gamma$. Let $(X, \Gamma)$ be a minimal flow. Suppose there is an $x \in X$ such that $x t$ is an $S$ almost periodic point for every $t \in \Gamma$. Then $(X, S)$ is minimal. 
Proof. Let $X_{0}=\overline{x S}$. Then $X_{0}$ is an $S$ minimal set. We show $X_{0}=X$. It is sufficient to show that $x \sigma s^{-1} \in X_{0}$, for all $s, \sigma \in S$. Clearly $x \sigma \in X_{0}$. Now $x \sigma s^{-1}$ is an $S$ almost periodic point, so $X_{1}=\overline{x \sigma s^{-1} S}$ is an $S$ minimal set. Then $x \sigma=x \sigma s^{-1} s \in X_{1}$. But $x \sigma \in X_{0}$ so $X_{1}=X_{0}$, and $x \sigma s^{-1} \in X_{0}$.

Theorem 2. Let $\Gamma$ be a group, let $S$ be a semigroup which generates $\Gamma$, and let $(X, \Gamma)$ be a minimal flow. Suppose that either $\Gamma$ is abelian, or $(X, \Gamma)$ is point distal. Then $(X, S)$ is minimal.

Proof. We show, in both cases, that there is an $x \in X$ such that $x t$ is an $S$ almost periodic point, for all $t \in T$, so the conclusion follows from Lemma 4 .

If $\Gamma$ is abelian, then $t \in \Gamma$ defines a flow automorphism of $(X, S)$. Hence if $x$ is $S$ almost periodic, so is $x t$.

Suppose $(X, \Gamma)$ is point distal, and let $x$ be a $\Gamma$ distal point. Then, if $t \in \Gamma, x t$ is a $\Gamma$ distal point, hence an $S$ distal point, and therefore an $S$ almost periodic point.

Let $\Gamma$ be a group and let $(X, \Gamma)$ be a flow. Let $\tilde{\Gamma}(X)$ be the group of homeomorphisms of $X$ defined by $\Gamma$.

Theorem 3. Let $\left(X_{i}, \Gamma\right)(i \in \mathcal{I})$ be a family of flows, and let $\Lambda$ be a subgroup of $\Gamma$. Suppose, for each $i \in \mathcal{I}, \tilde{\Gamma}\left(X_{i}\right)=\tilde{\Lambda}\left(X_{i}\right)$. Let $\left(x_{i}\right)$ be an almost periodic point of $\left(X_{i \in \mathcal{I}} X_{i}, \Lambda\right)$. Then $\left(x_{i}\right)$ is an almost periodic point of $\left(X_{i \in \mathcal{I}} X_{i}, \Gamma\right)$.

Proof. Recall that a point in a product flow is almost periodic if and only if its restriction to all finite products is almost periodic. Therefore it suffices to consider finitely many flows $\left(X_{i}, \Gamma\right)(i=1, \ldots, n)$ and an almost periodic point $\left(x_{1}, \ldots, x_{n}\right)$ in $\left(X_{1} \times \cdots \times X_{n}, \Lambda\right)$. We show that $\left(x_{1}, \ldots, x_{n}\right)$ is almost periodic for the action of $\Gamma$.

The proof is by induction on $n$. The case $n=1$ is an immediate consequence of the hypothesis. Suppose the result holds for $n-1$. Let $\left(x_{1}, x_{2}, \ldots, x_{n}\right)$ be a $\Lambda$ almost periodic point, and let $C$ be a maximal almost periodic set (with respect to $\Lambda)$ containing $\left(x_{1}, \ldots, x_{n}\right)$.

Let $z=\left(z_{1}, \ldots, z_{n}\right) \in\left(X_{1} \times \cdots \times X_{n}\right)^{|C|}$ with $\operatorname{range}(z)=C$. Then $z$ is a $\Lambda$ almost periodic point.

It follows from the induction hypothesis that $\left(z_{1}, \ldots, z_{n-1}\right)$ is a $\Gamma$ almost periodic point.

(Note that whenever $\tilde{\Gamma}(X)=\tilde{\Lambda}(X)$, then $\tilde{\Gamma}\left(X^{a}\right)=\tilde{\Lambda}\left(X^{a}\right)$ for any cardinal number $a$.)

Let $A_{i}=\pi_{i}(C)$ (where $\pi_{i}$ is the projection of $X_{1} \times \cdots \times X_{n}$ onto $X_{i}$ ). Then $A_{i}$ is a maximal almost periodic set for both the $\Lambda$ and $\Gamma$ actions.

Let $\left(z_{1}, \ldots, z_{n-1}, z_{n}^{\prime}\right) \in \overline{\left(z_{1}, \ldots, z_{n}\right) \Gamma}$ be an almost periodic point for both the $\Gamma$ and $\Lambda$ actions. Consider the point $\left(z_{1}, \ldots, z_{n-1}, z_{n}^{\prime}, x_{n}\right)$. Note that $\left(z_{1}, \ldots, z_{n-1}, x_{n}\right)$ is a $\Lambda$ almost periodic point. Therefore, there is a $\Lambda$ almost periodic point of the form $\left(z_{1}, \ldots, z_{n-1}, z_{n}^{\prime \prime}, x_{n}\right) \in \overline{\left(z_{1}, \ldots, z_{n-1}, z_{n}^{\prime}, x_{n}\right) \Lambda}$. Now range $\left(z_{n}^{\prime \prime}\right)$ is a maximal almost periodic set in $X_{n}$ so $x_{n} \in \operatorname{range}\left(z_{n}^{\prime \prime}\right)$. Also, $\left(z_{1}, \ldots, z_{n-1}, z_{n}^{\prime \prime}\right)$ is a $\Gamma$ almost periodic point (since it is in the orbit closure of the $\Gamma$ almost periodic point $\left.\left(z_{1}, \ldots, z_{n}^{\prime}\right)\right)$. Since $x_{i} \in \operatorname{range}\left(z_{i}\right)$ for $i=1, \ldots, n-1$, it follows that $\left(x_{1}, \ldots, x_{n}\right)$ is $\Gamma$ almost periodic.

With appropriate modifications, Theorem 2 can be stated and proved in case $\Gamma$ and $\Lambda$ are semigroups. 
The following is a (slight) generalization of a theorem of Glasner [G]. (Glasner assumed minimality of the action of $T$.)

Theorem 4. Let $T$ be a homeomorphism of the compact space $X$, let $n$ be a positive integer, and let $\theta$ and $\tau$ be the homeomorphisms of $X^{n}=X \times \cdots \times X$ defined by $\theta=T \times \cdots \times T$, and $\tau=T \times T^{2} \times \cdots \times T^{n}$. Let $x=\left(x_{1}, \ldots, x_{n}\right)$ be an almost periodic point for $\theta$. Then $x$ is an almost periodic point for the group generated by $\theta$ and $\tau$.

Proof. Let $\Lambda=\mathbf{Z}$, and let $\Gamma$ be the direct product of $\Lambda$ and $\mathbf{Z}^{n}=\mathbf{Z} \times \cdots \times \mathbf{Z}$. We may regard $\Lambda$ as a subgroup of $\Gamma$. Let $X_{i}=X$ for $i=1, \ldots, n$. The $\Lambda$ action on $X_{i}$ is by the homeomorphism $T$, and the action of $\mathbf{Z}^{n}$ on $X_{i}$ is defined by $\left(k_{1}, \ldots, k_{n}\right)(x)=T^{i k_{i}}(x)$. It is clear that $\tilde{\Lambda}\left(X_{i}\right)=\tilde{\Gamma}\left(X_{i}\right)$ and the result follows from Theorem 3 .

We are indebted to Benjamin Weiss for pointing out the following example of a minimal action of a group with a generating subsemigroup whose action is not minimal. Let $\Gamma=F_{2}$, the free group on two generators $a$ and $b$, and let $X$ be the space of infinite one-sided sequences $x=x_{1} x_{2} \ldots$ with $x_{i} \in\left\{a, b, a^{-1}, b^{-1}\right\}$ such that $x_{i} x_{i+1}$ is not of the form $a a^{-1}, a^{-1} a, b b^{-1}, b^{-1} b$. Let $\Gamma$ act on $X$ (on the left) by concatenation, and cancellation if required. (For example, if $x=a^{-1} a^{-1} b b a \ldots$, $b x=b a^{-1} a^{-1} b b a \ldots$ and $a x=a^{-1} b b a \ldots$ ) It is easy to see that this action of $F_{2}$ is minimal. Now let $S$ be the subsemigroup of $\Gamma$ generated by $a$ and $b$, and $E$ the closed subset of $X$ consisting of points in which only $a$ and $b$ appear. Then $S$ generates $\Gamma$, and $E$ is invariant under the $S$ action, so $(X, S)$ is not minimal.

A slight modification of this example shows that even for a semigroup of homeomorphisms, it is possible for the orbit closure of a point to be minimal, although the point is not in the minimal set. Let $X, E$, and $S$ be as above, and let $R=S a$. Then $E$ is minimal under the action of the semigroup $R$. If $x=a^{-1} b b b b \ldots$, then $\overline{R x}=E$ but $x \notin E$.

\section{REFERENCES}

[G] E. Glasner, Structure theory as a tool in topological dynamics, Descriptive set theory and dynamical systems, LMS Lecture Note Series 277, Cambridge University Press, 2000, 173209. MR 2001j:37020

Department of Mathematics, City College of New York, New York, New York 100319100

E-mail address: etkcc@cunyvm.cuny.edu

Department of Mathematics, University of Maryland, College Park, Maryland 20742-0001

E-mail address: jna@math.umd.edu 